九州大学学術情報リポジトリ

Kyushu University Institutional Repository

\title{
A Simple Method of Optical Evaluation of the Distribution of Muscle Extracellular Matrix by Immunofluorescence and Image Analysis using Fluorescent Semiconductor Nanocrystals (quantum dot)
}

Nakamura, Yoshi-Nori

Kuju Agricultural Research Center, Faculty of Agriculture, Kyushu University

Fumita, Tomiko

Kuju Agricultural Research Center, Faculty of Agriculture, Kyushu University

Hayashi, Ke isuke

Kuju Agricultural Research Center, Faculty of Agriculture, Kyushu University

Ebara, Fumio

Kuju Agricultural Research Center, Faculty of Agriculture, Kyushu University

他

https://doi.org/10.5109/14045

出版情報：九州大学大学院農学研究院紀要. 54 (1)，pp.103-108，2009-02-27. Faculty of Agriculture, Kyushu University

バージョン：

権利関係 : 


\title{
A Simple Method of Optical Evaluation of the Distribution of Muscle Extracellular Matrix by Immunofluorescence and Image Analysis using Fluorescent Semiconductor Nanocrystals (quantum dot)
}

\author{
Yoshi-Nori NAKAMURA ${ }^{1}$, Tomiko FUMITA ${ }^{1}$, Keisuke HAYASHI ${ }^{1}$, Fumio EBARA ${ }^{1}$, \\ Tetsuji ETOH $^{1}$, Yuji SHIOTSUKA ${ }^{1}$, Masa-aki HATTORI ${ }^{2}$ \\ and Takafumi GOTOH ${ }^{1, *}$
}

\author{
*Correspondent footnote Mailing address: Kuju Agricultural Research Center, \\ Faculty of Agriculture, Kyushu University, Oita 878-0201, Japan \\ (Received October 5, 2008 and accepted December 5, 2008)
}

\begin{abstract}
A simple method using optically stable quantum dots (Qdots) for immunohistochemical (IHC) analysis was tested to investigate their signal localization and stability in extracellular matrix (ECM) components of bovine longissimus thoracis muscles in fattened Japanese black steers. Detection of primary antibodies of each ECM component in IHC was based on fluorescence from streptavidin-linked inorganic crystals of cadmium selenide in Qdots with a fluorescence microscope (FM). We could observe each ECM component by IHC using Qdots in a FM for a long time. Each ECM component was detected clearly in the perimysium and endomysium in skeletal muscle. Using computer image analysis software, each ECM component was reconstructed in detail: structures were clear, with the distribution of each component of the ECM shown after 3D conversion based on the strength of fluorescenece. It is possible and useful to apply a simple method of IHC using Qdots under FM to the detailed investigation of ECM components in skeletal muscle.
\end{abstract}

Keywords: extracellular matrix, muscle, quantum dots, reconstruction

\section{INTRODUCTION}

The connective tissue in bovine skeletal muscle is composed of extracellular matrix (ECM) such as collagen, fibronectin, laminin and decorin (Nishimura, Futami, Taneichi, Mori \& Hattori, 2002; Nishimura, Ojima, Hattori \& Takahashi, 1997). In particular, the collagen is the major protein in skeletal muscle (Purslow, 2002), and various observation methods such as immunohistochemistry (IHC) (Listrat, Picard \& Geay, 1999; Nakamura, Iwamoto, Tabata \& Ono, 2003a) and cellmaceration/scanning electron microscopy (SEM) have been used (Ohtani, Ushiki, Taguchi \& Kikuta, 1988; Nakamura, Iwamoto, Shiba, Miyachi, Tabata \& Nishimura, 2004a). However, the functional roles and tissue construction of ECM in skeletal muscle have been poorly understood because of its complex matrix. We have previously reported collagen fiber networks in skeletal muscle of pig (Nakamura, Iwamoto, Ono, Shiba, Nishimura \& Tabata, 2003b) and chicken (Nakamura, Iwamoto, Shiba, Miyachi, Tabata \& Nishimura, 2004b) using a cell-maceration/SEM method in addition to a non-fluorescence IHC method. It was found that collagen fiber networks in domestic animals change with growth (Nakamura et al., 2004a), muscle fiber type (Nakamura et al., 2003a) and muscle component

Kuju Agricultural Research Center, Faculty of Agriculture, Kyushu University, Oita 878-0201, Japan

${ }^{2}$ Laboratory of Reproductive Physiology and Biotechnology, Faculty of Agriculture, Kyushu University, Fukuoka 812-8581, Japan

* Corresponding author (E-mail: gotoh@farm.kyushu-u.ac.jp)
(Nakamura et al., 2003b). However, the cell-maceration/SEM method makes it impossible to identify collagen types in addition to other ECM components because of the use of an alkaline solution in the preparation of the collagen specimens. Therefore we need to incorporate other methods to investigate the structure of ECM components in skeletal muscle in detail.

In our previous reports (Nakamura et al., 2007a,b), we demonstrated a three-dimensional reconstruction of intramuscular collagen networks of bovine muscle using IHC and confocal laser scanning microscope (CLSM). Generally, the fluorescence markers such as fluorescein isothiocyanate (FITC) and rhodamine B isothiocyanate (RITC) are used in IHC to reconstruct target proteins by CLSM and image analysis. We investigated the changes of connective tissue component of Masseter (MA) muscle in steers in concentrate- (C) and roughage-fed (R) groups (Nakamura et al., 2007a). We reconstructed muscle collagen networks using antibovine collagen types I, III, IV, V, VI, fibronectin and laminin polyclonal antibodies and FITC by CLSM and image analysis. The three-dimensional reconstructed images of connective tissue component of MA muscle of group $\mathrm{C}$ and $\mathrm{R}$ was three-dimensionally reappeared by immunohistochemical/confocal laser-scanning microscopy. Connective tissue component which composed in perimysium of group $\mathrm{C}$ and $\mathrm{R}$ was observed in plate-shaped layers. Type III collagen and fibronectin were threedimensionally and strongly observed in perimysium and endomysium in group R. On the other hand, honeycomb-shaped connective tissue component in endomysium surrounded muscle fibers. These results indicated that there were different developmental changes among 
connective tissue component in MA muscle by exercise (masticating), and the immunohistochemical/confocal laser-scanning microscope method was quite useful to investigate a structural relation among connective tissue component in skeletal muscle. However, there are problems of a short observation period due to fading of fluorescence markers as well as the complicated operation of CLSM. In particular, long observation times are required for looking and taking a photograph of each ECM component in the same location using muscle serial sections because there is a difference in the localization of each ECM component in the perimysium and endomysium in skeletal muscle (Nakamura et al., $2007 a, b)$. It is difficult to identify the same position for observation in each muscle serial section in addition to making changes in magnification.

Quantum dots (Qdots) were produced as a new tool in life sciences because of their interesting and unique optical properties (Michalet et al., 2005). Qdots are fluorescent semiconductor nanocrystals (Alivisatos, 1996), and have various sizes tailored to different wavelengths for example Qdots 510 650 (Jaiswal \& Simon, 2004). Qdots are used as fluorescent tags in various research fields, for example chemistry, biochemistry and molecular biology (Howarth, Takao, Hayashi \& Ting, 2005; Jaiswal, Mattoussi, Mauro \& Simon, 2003; Müller, Houben, Barker, Xiao, Käs \& Melzer, 2006) in addition to three-dimensional imaging analysis by CLSM (Ferrara et al., 2006; Matsuno, Itoh, Takekoshi, Nagashima \& Osamura, 2005).

Actually, to keep CLSM might be very costly in normal laboratories. We need the better way to understand functional distributions of ECM by a simpler way. We have tried to develop a simple technique for reconstruction of bovine muscle ECM components using Qdots, which radiate for a long time, a normal fluorescence microscope (FM), and computer image analysis software which makes the structures clear, showing the distribution of each ECM component by 3D conversion based on the strength of fluorescence. The purpose of this study was to clarify ECM distributions of bovine muscles and intramuscular fat tissues by this simple method.

\section{MATERIALS AND METHODS}

\section{Tissue preparation}

Fattened Japanese black steers (castrated males, $n=7,792.1 \pm 6.8$ days of age, $357.4 \pm 25.5 \mathrm{~kg}$ of body weight) were used. The steers were slaughtered in the Oita district meat center (Oita, Japan), and muscle samples were collected from their right side. The longissimus thoracis (LT) muscles were excised from the carcasses which were stored at $4^{\circ} \mathrm{C}$ for 1 week in the Oita district meat center. These materials, in about $1 \mathrm{~cm}^{3}$ blocks for immunohistochemical observation, were freshly dissected from the center of the LT muscle and rapidly frozen with a Tissue-Tek O.C.T. compound (Sakura Finetek U.S.A., Torrance, CA, USA) in liquid nitrogen. Tissue samples were stored at $-40{ }^{\circ} \mathrm{C}$ until hematoxylin-eosin
$(\mathrm{H}-\mathrm{E})$ and oil red staining and IHC analysis.

\section{IHC using Qdots}

Transverse non-fixed frozen and serial sections (8 $\mu \mathrm{m}$ thickness) were prepared using a cryostat (CM 1850, Leica; Leica Instruments GmbH, Nussloch, Germany) at $-20^{\circ} \mathrm{C}$. The sections were mounted on glass slides previously coated with $10 \%$ poly-L-lysine solution (Sigma-Aldrich, St. Louis, MO, USA) in distilled water. The tissue sections on glass slides were surrounded by a liquid blocking pen (immEdge hydrophobic barrier pen, H-4000; Vector Laboratories, Burlingame, CA, USA). The sections were fixed in acetone for $5 \mathrm{~min}$ at $4{ }^{\circ} \mathrm{C}$. After three washes in phosphate buffered saline (PBS) for 5 min at room temperature, the sections were treated with $0.3 \%$ hydrogen peroxide in methanol for $20 \mathrm{~min}$ at room temperature, and then washed with PBS. After these pre-treatments with $1 \%$ bovine serum albumin (Lot no. 18555; Rockland; Gilbertsville, PA, USA) and 1\% normal goat serum blocking solution (Lot no. 60782716; Zymed Laboratories; South San Francisco, CA, USA) in PBS (blocking buffer) for 20 min at room temperature, the sections were stained by primary antibodies for $2 \mathrm{~h}$ at room temperature. We used rabbit anti-bovine collagen types I, III, IV, V and VI polyclonal antibodies (Lot no. LB-1197, 1387, 1407, 1597 and 1697; LSL Co., Ltd., Princeton, NJ, USA), rabbit anti-bovine fibronectin polyclonal antibody (Lot no. LB-1027; LSL) and rabbit anti-mouse laminin polyclonal antibody (Lot no. LB-1013; LSL), both diluted 1:500 with PBS, as primary antibodies, and only PBS for negative control. Non-cross-reactions among these antibodies had been already confirmed and proved by LSL. After washing with PBS, the sections were treated by biotinylated goat anti-rabbit immunoglobulin G (IgG) as a secondary antibody, followed by avidin-biotin conjugate (ABC) reagents (Vectastain ABC rabbit IgG kit; Lot no. PK-6101; Vector Laboratories) for $30 \mathrm{~min}$ at room temperature. After washing with PBS, the sections were incubated in Qdot streptavidin conjugate solution (Qdot 525 streptavidin conjugate, starter kit; dilution 1:100 with blocking buffer; Lot no. Q10143MP; Invitrogen, Carlsbad, CA, USA) for $30 \mathrm{~min}$ at room temperature in a box shielded from light. All antibodies and Qdot streptavidin conjugates were centrifuged at $2000 \times \mathrm{g}$ for $1 \mathrm{~min}$ before use to remove aggregates. After washing with PBS, the sections were mounted in an enclosure solution (Mountquick, aqueous; Daido Sangyo, Saitama, Japan).

\section{Image analysis}

The observations of the sections were carried out on a FM (Microphot-FXA; Nikon, Tokyo, Japan) with phase contrast method, photographed by a digital camera (color camera system MP5Mc/OL; Olympus, Tokyo, Japan) and computer image analysis software (Q Capture PRO version 5.1; Qimaging, Surrey, Canada) used to clarify fluorescence intensities. Images were collected on a FM using a filter cassette (B-2A, 510 dichroic mirror, 450 490 excitation filters, 520 emission filter, Nikon). Fluorescence images were background- 
corrected using computer image analysis software (Q Capture PRO version 5.1). We made a new image from a permeated image and fluoresced image in the Background Subtraction command in this software to compare the active image to the background image, and pixels deemed to be part of the background. The algorithm is based on the following formula: $C I_{x y}=I_{x y}-$ $B I_{x y}+M . I_{x y}$ is a pixel value of the original image at location $(\mathrm{x}, \mathrm{y}), B I_{x y}$ is a pixel value of the background image at location (x, y), $M$ is the average pixel value of the background image and $C I_{x y}$ is the new pixel value in the corrected image (Qimaging).

Next, the reconstruction of each ECM component was demonstrated by other computer image analysis software (Win ROOF version 5.0; Mitani Co, Tokyo, Japan). Original images were separated in red-greenblue (RGB), and reconstructed by three-dimensional display, especially 'rainbow 16' (Win ROOF).

\section{RESULTS AND DISCUSSION}

The images of each ECM component in bovine LT muscle, especially perimysium and endomysium, by IHC staining using Qdots are shown in Figs. 1 and 2. In this study, we used the same conditions for dilution and magnification of primary antibodies (1:500) and Qdot 525 streptavidin conjugated solutions (1:100) in addition to a 15 second exposure in a FM. As a result, all antibodies were clearly observed in the connective tissue. In detail, type I collagen was observed mainly in the perimysium (Fig. 1c). Type III, V and VI collagen were observed in the perimysium and endomysium (Figs. 1e, 2a and 2c). Type IV collagen and laminin were observed mainly in the endomysium (Figs. 1g and $\mathbf{2 g}$ ). On the other hand, fibronectin was observed straggly in the perimysium and endomysium (Fig. 2e). Localizations of ECM components in bovine LT muscle in this study were similar to that of another report using bovine semitendinosus muscle (Nishimura et al., 1997). Until now, we have used another IHC method to observe ECM components in skeletal muscle (Nakamura et al., 2003b, 2004b). This method used a combined peroxidase which was developed with a substrate consisting of diaminobenzidine tetrahydrochloride. In the present study, we used a new IHC method using Qdots and image analysis to clearly study ECM components in skeletal muscle. In fact, very clear images of each ECM component were obtained by image analysis (Figs. 1d, 1f, 1h, 2b, 2d, 2f and $\mathbf{2 h}$ ). These images were obtained by phase contrast and fluorescence microscopic photographs using the image analysis software. Respective serial section which had been immunohistochemically stained by each antibody was used for FM observation. First, using phase contrast, a specific and common location for observation was decided on these serial sections. Next, by changing to filter for fluorescence antibody, each ECM component was observed in same section. Finally, a photo montage was compiled from phase contrast and fluorescence images using computer image analysis software (Q Capture
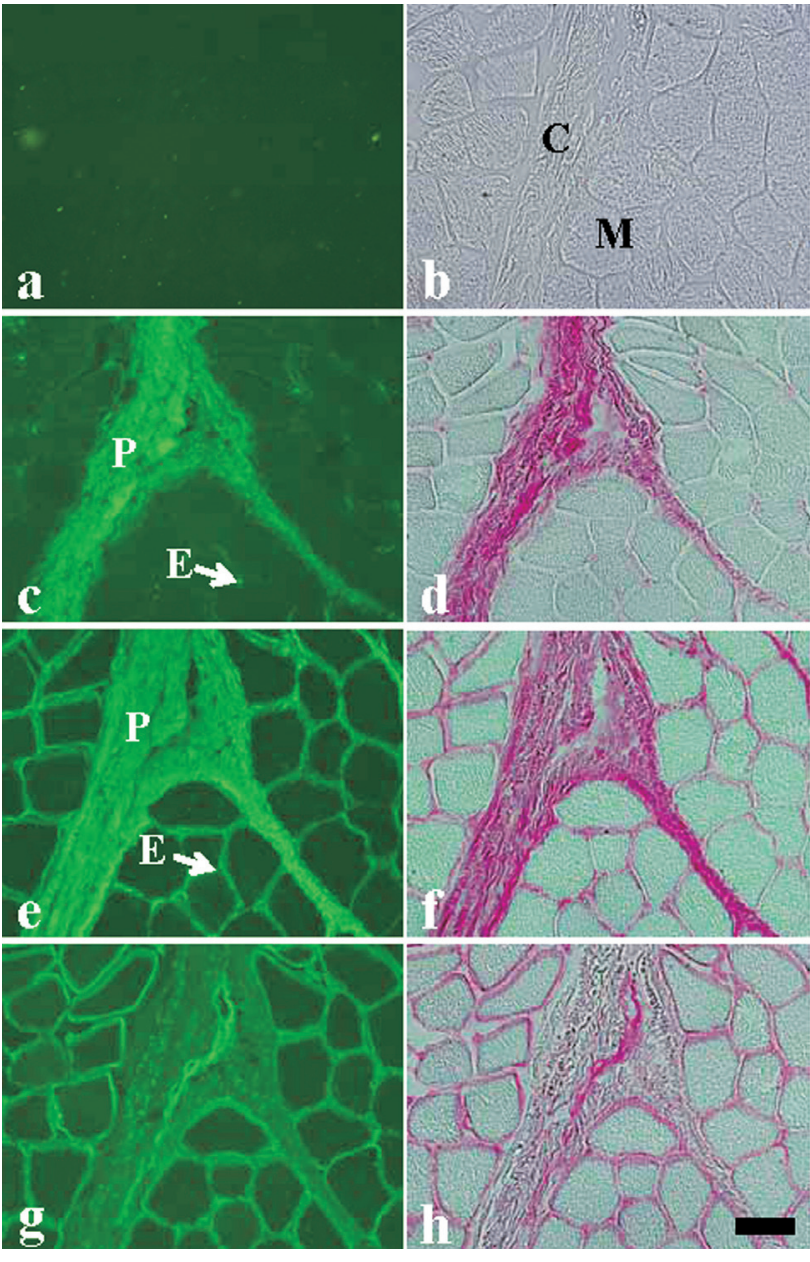

Fig. 1. Localization of extracellular matrix (ECM) components in bovine skeletal muscle. The transverse non-fixed frozen and serial sections ( $8 \mu \mathrm{m}$ thickness) of longissimus thoracis (LT) muscles were stained with the immunohistochemical (IHC) method using anti-bovine collagen type I (c), type III (e) and type IV (g) antibodies by a combination of an anti-rabbit immunoglobulin G biotin/quantum dots (Qdot) 525 streptavidin conjugate, and only PBS for negative control (a). In particular, phase contrast by control section incubated without the primary antibody was shown in Fig. 1b. Non-cross-reactions among these antibodies had been already confirmed and proved by LSL Co. Ltd. (Princeton, NJ, USA). Photo montages, type I (d), type III (f) and type IV (h) collagens, were compiled from phase contrast (data not shown) and fluorescence images (c, e and g) using computer image analysis software (Q Capture PRO Version 5.1), respectively. Photo montage by computer image analysis was possible to do detailed observations on each tissue section in same location in spite of subtle gaps of serial sections. C: connective tissue; E: endomysium; M: muscle fiber; P: perimysium, respectively. Bars: $50 \mu \mathrm{m}$.

PRO Version 5.1). Therefore, it was possible to do detailed observations on each tissue section in same location. However, in this study, the one tissue section was used for one antibody, for example type I collagen or type III collagen. Therefore the multiplex dyeing by the IHC method using other Qdots, for example Qdot $565,585,605$ and 655 corresponding to different wavelengths, is needed to investigate the location of each antibody on the same tissue section. In all antibodies 


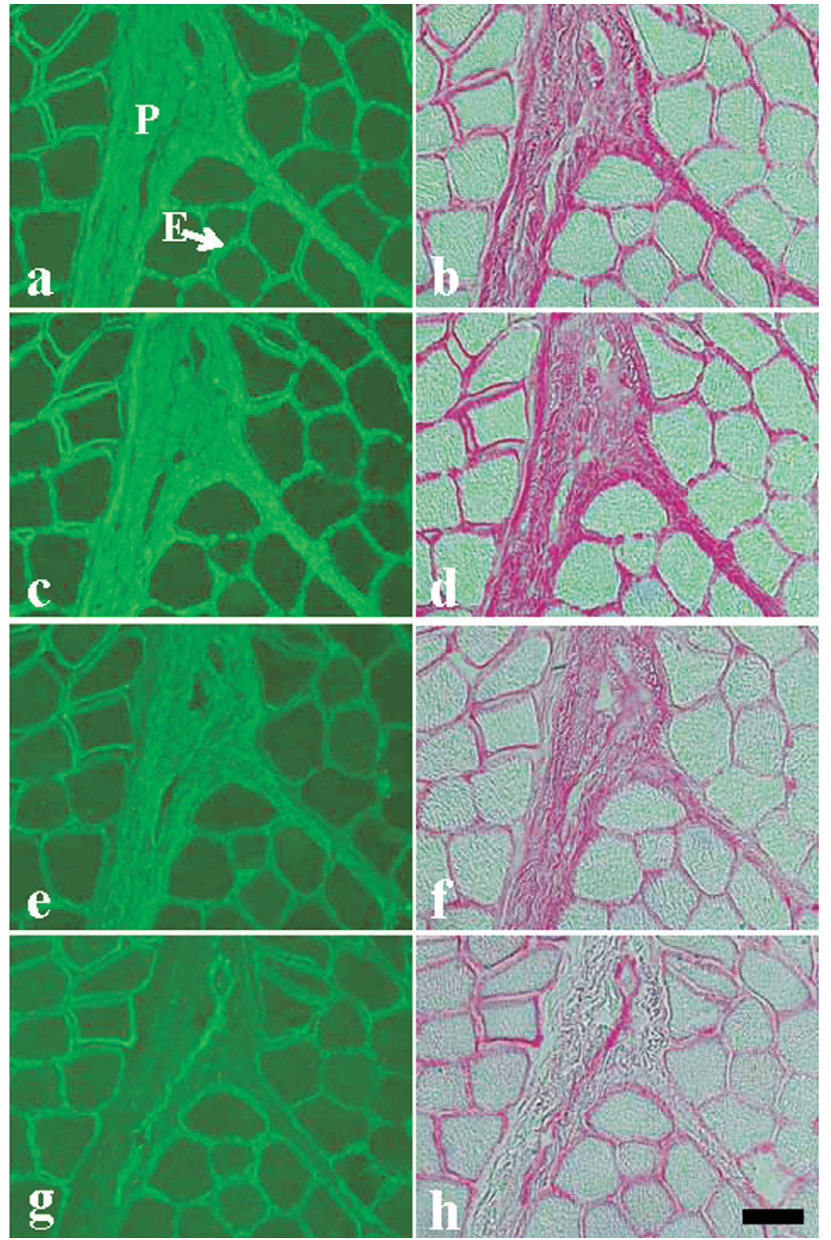

Fig. 2. Localization of ECM components in bovine skeletal muscle. The transverse non-fixed frozen and serial sections (8 um thickness) of LT muscles were stained with the IHC method using anti-bovine collagen type V (a), type VI (c) collagens, fibronectin (e) and laminin (g) antibodies by a combination of an anti-rabbit immunoglobulin G biotin/ Qdot 525 streptavidin conjugate. Non-cross-reactions among these antibodies had been already confirmed and proved by LSL. Photo montages, type V (b), type VI (d) collagens, fibronectin (f) and laminin (h), were compiled from phase contrast (data not shown) and fluorescence images (a, c, e and g) using computer image analysis software (Q Capture PRO Version 5.1), respectively. E: endomysium; P: perimysium, respectively. Bars: $50 \mu \mathrm{m}$.

used in this study, non-cross-reactions among them have been confirmed. In addition, we set up a $15 \mathrm{sec}-$ ond exposure to uniformalize the strong and weak levels of light of each antibody. As a result, we could clearly observe the localization of each antibody of the ECM components in the phase contrast images. However, we could not quantify each ECM component in bovine LT muscles by image analysis in this study. Therefore we consider that another method, for example western blot analysis, should be used to quantify each ECM component in skeletal muscles. Reconstructed images of each ECM component in LT muscle, especially perimysium and endomysium are shown in Fig. 3. Each ECM component observed in the perimysium was three-dimensionally reconstructed as plate-shaped layers whereas each ECM component observed in the endomysium by
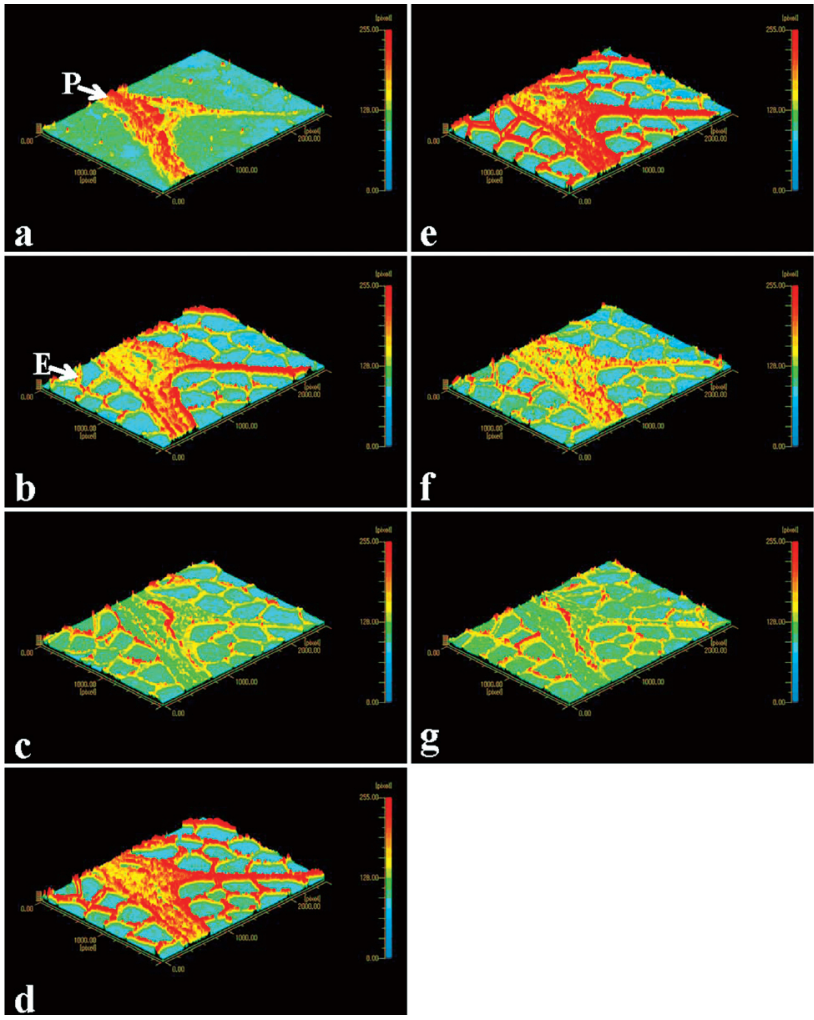

Fig. 3. Three-dimensional (3D) reconstruction images of ECM components in bovine LT muscles by image analysis (Win ROOF). Type I (a), type III (b), type IV (c), type V (d), type VI (e) collagens, fibronectin (f) and laminin (g), respectively. E: endomysium; P: perimysium, respectively Magnification of images was equal for each image corresponding to each ECM component.

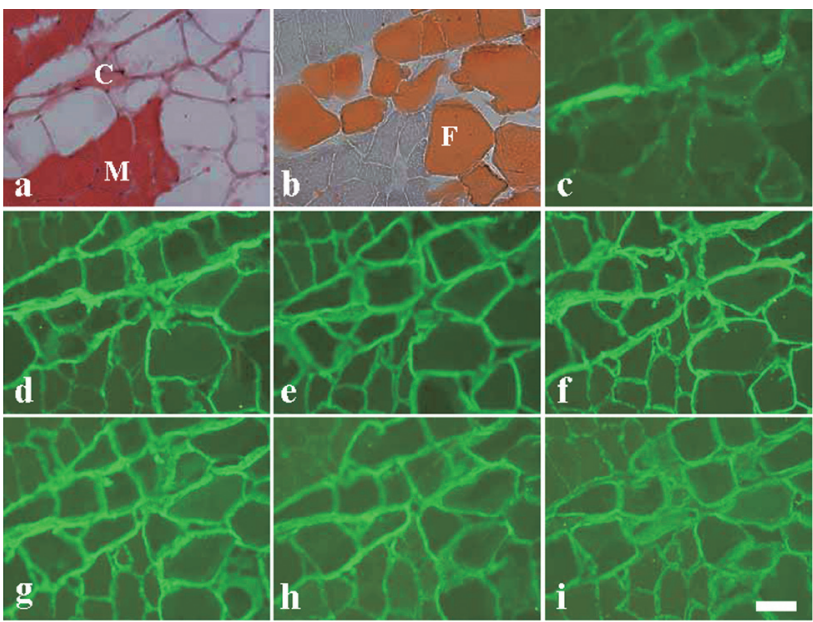

Fig. 4. Localization of ECM components, especially surrounded with fat tissue, in bovine skeletal muscle. The transverse non-fixed frozen and serial sections ( $8 \mu \mathrm{m}$ thickness) of LT muscles were stained with the IHC method using antibovine collagen type I (c), type III (d), type IV (e), type V (f), type VI (g) collagens, fibronectin (h) and laminin (i) antibodies by a combination of an anti-rabbit immunoglobulin G biotin/Qdot 525 streptavidin conjugate in addition to hematoxylin-eosin (a) and oil red (b) staining. Noncross-reactions among these antibodies had been already confirmed and proved by LSL. C: connective tissue; F: fat tissue; M: muscle fiber, respectively. Bars: $50 \mu \mathrm{m}$. 
FM was surrounded by myofibers. In a previous report (Nakamura et al., 2007a,b), we used CLSM to observe each ECM component. However, in this study, very clear images of each ECM component were reconstructed in spite of using FM. We think that it is possible to do reconstruction with small particles of Qdot under the resolving power of an optical microscope. However, in this study, a tissue thickness under $10 \mu \mathrm{m}$ was suitable. Thicker tissue sections, for example $40 \mu \mathrm{m}$, were not suitable as the rugged surface of the tissue section, especially transformation of the perimysium, in FM had a low depth of focus (data is not shown).

ECM components surrounding fat tissue were observed by the same method in this study (Figs. 4 and 5). Each ECM component was assembled in a thin membranous structure similar to the endomysium (Fig. 4), however, reconstructed images were assembled continuously in comparison with the endomysium (Fig. 5). For example, a basement membrane composed of type IV collagen and laminin was thicker than the surrounding endomysium (Figs. 5c and g). In addition, fibronectin was observed in a continuous plate-layer in the surrounding fat tissue (Fig. 5f) in comparison with that of the endomysium (Fig. 3f). These results indicate that there is a different construction of ECM components in the surrounding muscle fiber and fat tissue.

We would like to emphasize the differences in obser-

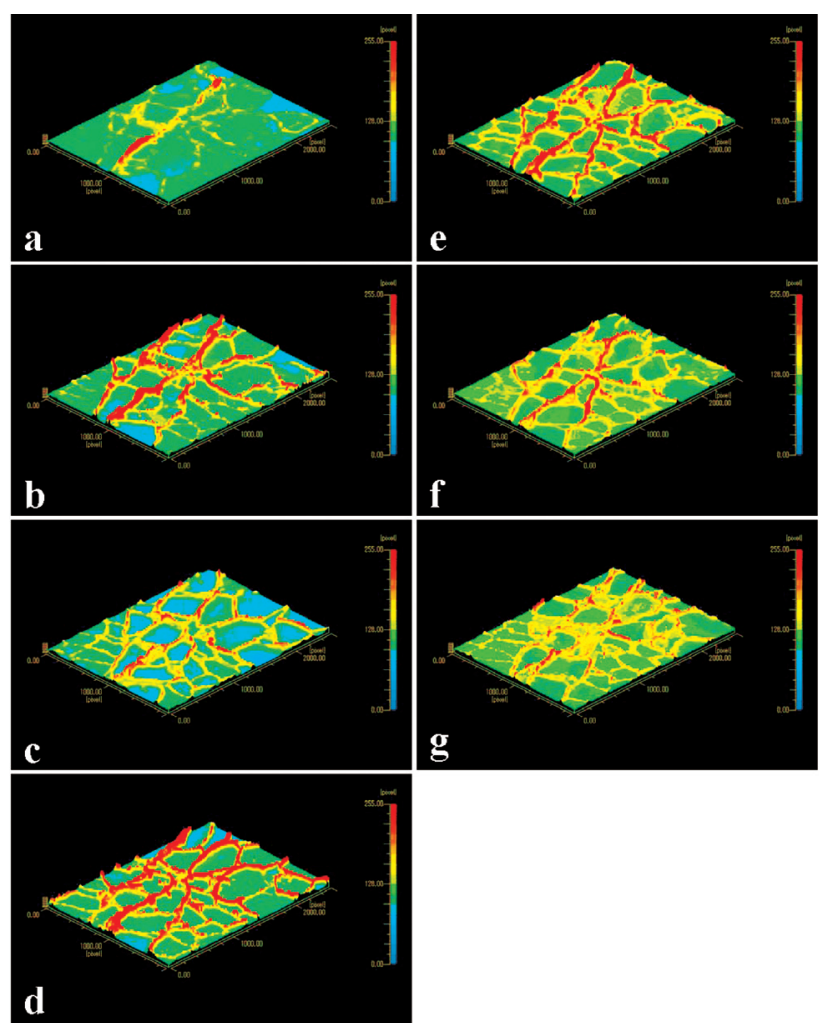

Fig. 5. 3D reconstruction images of ECM in bovine LT muscles, especially ECM components surrounded with fat tissue, by image analysis (Win ROOF). Type I (a), type III (b), type IV (c), type V (d), type VI (e) collagens, fibronectin (f) and laminin (g), respectively. Magnification of images was equal for each image corresponding to each ECM component. vation time in comparison with the normal IHC method. Generally, an IHC method by a FM uses various fluorescence coloring substances such as a FITC and RITC. However, these coloring substances fade immediately after excitation. We used an antibody of type $\mathrm{V}$ collagen to investigate the observation time of an IHC method using Qdots. In this study, we set up $30 \mathrm{~min} / \mathrm{d}$ observation and 15 seconds of exposure by a FM. In addition, tissue samples were shielded from light until the next observation. As a result, we could observe type V collagen up to $120 \mathrm{~h}$ later (data is not shown). Our result of a prolonged observation period in this study is similar to other reports (Müller et al., 2006). However, the darkness gradually increased, and the fading progressed here and there in the perimysium and endomysium. Therefore, the fact that there is the possibility of prolonged observation suggests that an observation time after excitation is needed to investigate each target protein.

In conclusion, we clarified detailed ECM distributions of bovine muscle and intramuscular fat tissues by our simple method. By proceeding with this investigation, we would understand complicated three-dimensional distributions of many kinds of ECM which were closely related to meat quality and toughness. Finally, we suggest that it is possible and useful to apply this simple method using Qdots-labeled IHC, a normal FM and computer image analysis software to the investigation of ECM components in bovine skeletal muscles.

\section{REFERENCES}

Alivisatos, A. P. 1996 Perspectives on the physical chemistry of semiconductor nanocrystals. Journal of Physical Chemistry, 100: 13226-13239

Ferrara, D. E., D. Weiss, P. H. Carnell, P. P. Vito, D. Vega, X. Gao, S. Nie and W. R. Taylor 2006 Quantitative 3D fluorescence technique for the analysis of en face preparations of arterial walls using quantum dot nanocrystals and two-photon excitation laser scanning microscopy. American Journal of Physiology. Regulatory, Integrative and Comparative Physiology, 290: R114-R123

Howarth, M., K. Takao, Y. Hayashi and A. Y. Ting 2005 Targeting quantum dots to surface proteins in living cells with biotin ligase. Proceedings of the National Academy of Sciences of USA, 102: 7583-7588

Jaiswal, J. K., H. Mattoussi, J. M. Mauro and S. M. Simon 2003 Long-term multiple color imaging of live cells using quantum dot bioconjugates. Nature Biotechnology, 21: 47-51

Jaiswal, J. K. and S. M. Simon 2004 Potentials and pitfalls of fluorescent quantum dots for biological imaging. TRENDS in Cell Biology, 14: 497-504

Listrat, A., B. Picard and Y. Geay 1999 Age-related changes and location of type I, III, IV, V and VI collagens during development of four foetal skeletal muscles of double-muscled and normal bovine animals. Tissue and Cell, 31: 17-27

Matsuno, A., J. Itoh, S. Takekoshi, T. Nagashima and R. Y. Osamura 2005 Three-dimensional imaging of the intracellular localization of growth hormone and prolactin and their mRNA using nanocrystal (quantum dot) and confocal laser scanning microscopy techniques. Journal of Histochemistry and Cytochemistry, 53: 833-838

Michalet, X., F. F. Pinaud, L. A. Bentolila, J. M. Tsay, S. Doose, J. J. Li, G. Sundaresan, A. M. Wu, S. S. Gambhir and S. Weiss 2005 Quantum dots for live cells, in vivo imaging, and diagnostics. Science, 307: 538-544 
Müller, F., A. Houben, P. E. Barker, Y. Xiao, J. A. Käs and M. Melzer 2006 Quantum dots - a versatile tool in plant science? Journal of Nanobiotechnology, 4: 5

Nakamura, Y.-N., H. Iwamoto, S. Tabata and Y. Ono 2003a Comparison of collagen fibre architecture between slowtwitch cranial and fast-twitch caudal parts of broiler M. latissimus dorsi. British Poultry Science, 44: 374-379

Nakamura, Y.-N., H. Iwamoto, Y. Ono, N. Shiba, S. Nishimura and S. Tabata 2003b Relationship among collagen amount, distribution and architecture in the $M$. longissimus thoracis and M. pectoralis profundus from pigs. Meat Science, $\mathbf{6 4}$ : $43-50$

Nakamura, Y.-N., H. Iwamoto, N. Shiba, H. Miyachi, S. Tabata and S. Nishimura 2004a Growth changes of the collagen content and architecture in the pectoralis and iliotibialis lateralis muscles of cockerels. British Poultry Science, 45: $753-761$

Nakamura, Y.-N., H. Iwamoto, N. Shiba, H. Miyachi, S. Tabata and S. Nishimura 2004b Developmental states of the collagen content, distribution and architecture in the pectoralis, iliotibialis lateralis and puboischiofemoralis muscles of male Red Cornish $\times$ New Hampshire and normal broilers. British Poultry Science, 45: 31-40

Nakamura, Y.-N., H. Iwamoto, T. Etoh, Y. Shiotsuka, T. Yamaguchi, Y. Ono, S. Tabata, S. Nishimura and T. Gotoh 2007a Three-dimensional observation of connective tissue of bovine masseter muscle under concentrate- and rough- age-fed conditions by using immunohistochemical/confocal laser-scanning microscopic methods. Journal of Food Science, 72: E375-E381

Nakamura, Y.-N., H. Iwamoto, T. Yamaguchi, Y. Ono, Y. Nakanishi, S. Tabata, S. Nishimura and T. Gotoh 2007b Threedimensional reconstruction of intramuscular collagen networks of bovine muscle: a demonstration by an immunohistochemical/confocal laser-scanning microscopic method. Animal Science Journal, 78: 445-447

Nishimura, T., K. Ojima, A. Hattori and K. Takahashi 1997 Developmental expression of extracellular matrix components in intramuscular connective tissue of bovine semitendinosus muscle. Histochemistry and Cell Biology, 107 215-221

Nishimura, T., E. Futami, A. Taneichi, T. Mori and A. Hattori 2002 Decorin expression during development of bovine skeletal muscle and its role in morphogenesis of the intramuscular connective tissue. Cells Tissues Organs, 171: 199-214

Ohtani, O., T. Ushiki, T. Taguchi and A. Kikuta 1988 Collagen fibrillar networks as skeletal frameworks: a demonstration by cell-maceration/scanning electron microscope method. Archives of Histology and Cytology, 51: 249-261

Purslow, P. P. 2002 The structure and functional significance of variations in the connective tissue within muscle. Comparative Biochemistry and Physiology Part A, 133 947-966 